\title{
Gyrotron Experiments Employing a Field Emission Array Cathode
}

\author{
M. Garven,* S. N. Spark, ${ }^{\dagger}$ A. W. Cross, S. J. Cooke, and A. D. R. Phelps \\ Department of Physics and Applied Physics, University of Strathclyde, Glasgow, G4 ONG, United Kingdom
} (Received 2 April 1996)

\begin{abstract}
The design and operation of a field emission array (FEA) cathode and the subsequent demonstration of the first FEA gyrotron are presented. Up to $10 \mathrm{~mA}$ from 30000 tips was achieved reproducibly from each of ten chips in a gyrotron environment, namely, a vacuum $1 \times 10^{-8}$ mbar, $-50 \mathrm{kV}$ potential with multiple chip operation. The design parameters of the FEA gun were similar to those of a magnetron injection gun with an achievable electron beam current of 50-100 mA and measured power $720 \mathrm{~W} \mathrm{cw}$. Coherent microwave radiation was detected in both $\mathrm{TE}_{02}$ at $30.1 \mathrm{GHz}$ and $\mathrm{TE}_{03}$ at $43.6 \mathrm{GHz}$, with a starting current of $1 \mathrm{~mA}$. [S0031-9007(96)00889-7]
\end{abstract}

PACS numbers: 84.40.Ik, 52.75.Va

Field emission arrays (FEAs), which are central to the new technology of vacuum microelectronics, are emerging as novel sources of electrons. Development of FEAs at research centers around the world is proceeding with applications in microwave devices and display technology [1-6] in which they provide a compact, controllable electron source. FEAs have the potential to revolutionize free-electron microwave devices, since electron emission can in principle be modulated by small voltages $(10 \mathrm{~V})$ to very high frequencies $(\mathrm{GHz})$ to effectively prebunch the electron beam for coherent microwave generation. Additionally, FEAs are "cold" emitters with a fast turn-on capability, require no heater current, and permit high current densities with spatial and temporal control. Their unique combination of properties makes possible smaller, lighter, and more efficient microwave devices. This possibility is realized in this work with the demonstration of an FEA cathode used in a millimeter wave device.

Vacuum microelectronics involves electron transport in vacuum via the use of modern semiconductor microfabrication technology [1]. First proposed over 25 years ago, thin-film field-emission technology has experienced a resurgence in interest as microfabrication technology has matured [7]. The first functioning micron scale field emission devices were made by Spindt [8] and continuous improvements to this process have been made with corresponding improvements in performance [2]. The use of FEAs in microwave power tubes was first discussed by Brodie and Spindt [4].

In a gyrotron, mm-wave generation is due to the cyclotron resonance maser (CRM) instability. A beam of relativistic electrons undergoing cyclotron motion in a uniform axial magnetic field interacts collectively with a propagating electromagnetic wave, such that energy transfer occurs. The cyclotron frequency $\left(f_{c}=\omega_{c} / 2 \pi\right)$ of the beam in a magnetic induction $B$ is given via

$$
\omega_{c}=\frac{e B}{\gamma m_{0}},
$$

where $\gamma=1+|e V| / m_{0} c^{2}$ for a beam accelerating po- tential difference $V$ and $e / m_{0}$ is the electron charge/mass ratio. The interaction frequency is determined by the cyclotron resonance condition $\omega=s \omega_{c}+k_{z} v_{z}$, for a wave of angular frequency $\omega$ and axial wave-vector component $k_{z}$, where $s=1$ for the fundamental interaction. In the case of the gyrotron, $k_{z} v_{z} \ll \omega$ so that the effect of a spread in axial velocity is minimal and the emitted radiation is only mildly Doppler up-shifted from the cyclotron frequency.

For the $\mathrm{TE}_{m n}$ or $\mathrm{TM}_{m n}$ mm-wave mode of a cylindrical waveguide, the wave dispersion relation $\omega^{2}=c^{2} k_{\perp}^{2}+$ $c^{2} k_{z}^{2}$ must also be satisfied, in which the cutoff frequency $\omega_{\text {co }}=c k_{\perp}$ is mode dependent. Therefore, the gyrotron interaction frequency occurs slightly above both the mode cutoff and cyclotron frequencies.

Gyrotrons developed previously at Strathclyde University have been based on both field-immersed, field emission plasma flare cathodes [9-11] and thermionic magnetron injection gun (MIG) type cathodes. Recent gyrotrons have employed a thermionic cathode with measured long pulse operation up to $100 \mu$ s at $1 \mathrm{MW}$ beam power and a conversion efficiency of $10 \%$ at $42 \mathrm{GHz}$ [12].

A third type of gyrotron based on a microelectronic FEA cathode is presented here. This was designed to be a low power, $\sim 1 \mathrm{~kW}$ electron beam system operated both $\mathrm{cw}$ and long pulse, $\gg 100 \mu \mathrm{s}$. Previously, shortpulsed plasma flare cold cathodes have been employed in gyrotrons, which produced up to $6 \mathrm{MW} \mathrm{mm}$-wave output at a pulse repetition frequency of $330 \mathrm{~Hz}$. This repetition rate was limited by the power supply energy delivery and switching capabilities of the high voltage pulsed system. Utilizing FEAs promises ultimate repetition frequencies during pulsed operation well beyond this, as modulation does not require the switching of the high voltage power supply.

The FEAs employed in this work were fabricated by GEC-Marconi Ltd. and consisted of silicon tips approximately 1-2 $\mu \mathrm{m}$ high with $10 \mathrm{~nm}$ tip radii, aligned beneath holes in an extraction grid electrode. The grids consist of a $0.5 \mu \mathrm{m}$ layer of niobium supported by 
an $\mathrm{SiO}_{2}$ insulating layer, in which the silicon emitters protrude through holes typically $2 \mu \mathrm{m}$ in diameter. Previous experiments demonstrated [13] that for arrays with up to 10000 tips, average emission currents of $1 \mu \mathrm{A}$ per tip could be obtained under ideal UHV conditions.

The key parameters for the FEA cathode, which were based on a thermionic MIG [12], were the annular cathode radius, $12.5 \mathrm{~mm}$, and the slope angle, $4^{\circ}$. These parameters were not changed throughout the course of the experiments. Previous studies [13] investigated various chip designs and the most suitable for a gyrotron application was determined in terms of optimum emission current. The final emitter chip design used was $2.25 \times 2.25 \mathrm{~mm}^{2}$ in area with five $6000 \mathrm{Si}$ tip arrays, mounted on a $6.6 \mathrm{~mm}$ square flat package ("flatpack"). The chip and flatpack dimensions along with the MIG parameters dictated that a maximum of ten chips would fit into the FEA gun geometry, Fig. 1. A current of $10 \mathrm{~mA}$ per chip was measured indicating that the FEA cathode could achieve total emission currents of up to $100 \mathrm{~mA}$ from the ten chips.

One of the main advantages of this new type of cathode over its traditional counterparts is in the ability to control the spatial distribution of individual components of the beam. This was achieved by individually addressing each package in the FEA cathode, accomplished by supplying a separate control voltage to the $\mathrm{Nb}$ grid connections on each chip, with a common Si tip cathode potential. Such control was also found necessary during multiple chip operation to permit optimum operation of each chip, due to the slight variation in the grid voltage required by each chip to operate at its maximum emission current. An electrically isolated, fiber optically controlled power supply unit with ten channels, all variable from 0 to $150 \mathrm{~V}$, was designed for this specialized operation.

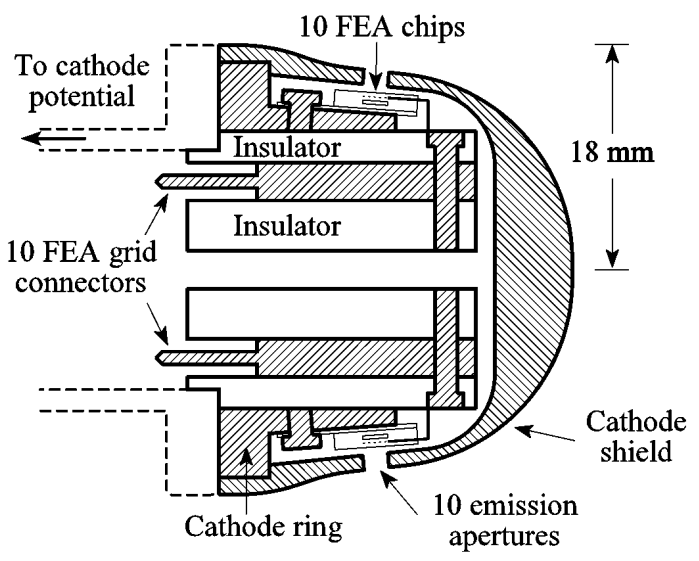

FIG. 1. Cross section through the head of the FEA cathode, with two of ten chips shown. For each chip, the silicon emitters are electrically connected to the common cathode ring via the flatpack base. Individual grid voltage control was achieved via microscopic bond wires to the package leads, which were electrically contacted to the grid connectors.
When mounted in the final electrode configuration the FEA cathode potential could be up to $-50 \mathrm{kV}$. With the vulnerable structure of the FEA chip, flatpack mounting and the $0.25 \mu \mathrm{m}$ bond wires exposed, the resulting electric field enhancement would cause catastrophic breakdown between the cathode components and other electrodes. To prevent this the chip mounting structure was surrounded by a field-relieving cathode shield, Fig. 1. To allow emitted electrons to propagate from the FEA cathode, ten holes, aligned with the FEAs, were made in the smooth contoured shield.

The FEA cathode emission currents are consistent with gyrotron starting currents, provided the gyrotron is operated with a high cavity $Q(>10000)$. Cavity inserts were employed to increase the cavity quality factor, $Q$, thereby reducing the starting current. For these experiments a dc central intracavity magnetic field, variable from 0 to $9 \mathrm{~T}$, was generated using a superconducting magnet which provided a uniform homogeneous magnetic field, varying by $<0.1 \%$ over a length of $10 \mathrm{~cm}$. The expected gyrotron operating characteristics were beam current $\sim 100 \mathrm{~mA}$, gating voltage $\sim 100 \mathrm{~V}$, accelerating potential $\sim 50 \mathrm{kV}$, emission area $\sim 1.5 \mathrm{~cm}^{2}$, annular beam distribution, operating vacuum $\sim 10^{-8}$ mbar with a cathode lifetime of $>100 \mathrm{~h}$, and both $\mathrm{cw}$ and pulsed operation.

The FEA cathode assembled in the gyrotron with the cathode and cavity magnetic field coils and cavity inserts is shown in Fig. 2. Electron trajectory modeling was used to verify the electron beam propagation from the FEA cathode to the interaction cavity. Thermal diagnostics were employed to detect the electron beam transport through the interaction region.

A standard UHV ceramic insulator was inserted in the electron beam transport tube, between the FEA anode and the gyrotron interaction cavity, to electrically isolate the cavity from the diode. The anode and cavity sections were separately connected to ground through two ammeters, allowing measurement of the electron return current from each section. Current reaching the cavity wall after passing through the interaction region was therefore measured directly, independently of the current flowing to the anode. In the experiment the anode current was minimized by optimizing the guiding

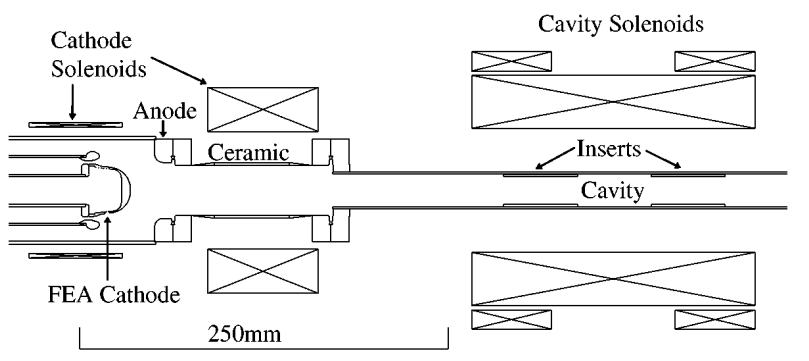

FIG. 2. Overview of the FEA gyrotron, showing the electrodes, magnetic field coils, ceramic insulator, and cavity components. 
magnetic fields. This provided a useful diagnostic by allowing direct detection of the current participating in the CRM interaction.

Figure 3 summarizes FEA cathode operation in terms of $I / V$ space, where $I$ in $\mathrm{mA}$ is the electron beam current measured to the gyrotron drift tube and $V$ in $\mathrm{kV}$ is the accelerating potential. The lower left hand corner sectioned off by the curved line represents the area where the FEA chips operated with minimum signs of stress, i.e., grid currents $\sim 100 \mu \mathrm{A}$ and emission currents $\sim 1 \mathrm{~mA}$. Chips were operated $\mathrm{cw}$ in this area for lifetimes of tens of hours with minimal degradation in performance. Typically a chip would operate only $30 \mathrm{~min}$ outside this area before showing signs of operational stress and increased grid currents.

There are two points which must be emphasized with respect to the future of FEAs in high power mmwave devices, (1) optimization of chip lifetime and (2) increase of beam power. The region of operation with optimized lifetime may be extended well beyond the upper half of Fig. 3 simply by operating multiple chips. This work suggests that chips of the design employed in this work will operate successfully with emission currents up to $\sim 6 \mathrm{~mA}$. Ten such chips would therefore supply $\sim 60 \mathrm{~mA}$, at $40 \mathrm{kV}$ to produce a $2.4 \mathrm{~kW}$ electron beam. This is a modest extrapolation of these results since both multiple chip operation and $\mathrm{cw} 10 \mathrm{~mA}$ operation from a single chip have been demonstrated.

In our experiments the maximum generated power was power supply limited since operating the FEAs outside the safe operation line resulted in increased grid currents. At any one time only three chips were operated which for the maximum power of $720 \mathrm{~W}$ represents an average of $6 \mathrm{~mA}$ per chip at $40 \mathrm{kV}$. The maximum measured beam power from a single FEA chip was $\sim 375 \mathrm{~W}$, emitting $9.37 \mathrm{~mA}$ with an accelerating potential of $40 \mathrm{kV}$, corresponding to a current density of $\sim 0.5 \mathrm{~A} / \mathrm{cm}^{2}$.

To verify that the electron beam passed through the cavity region, thermistor heat sensors were attached to the

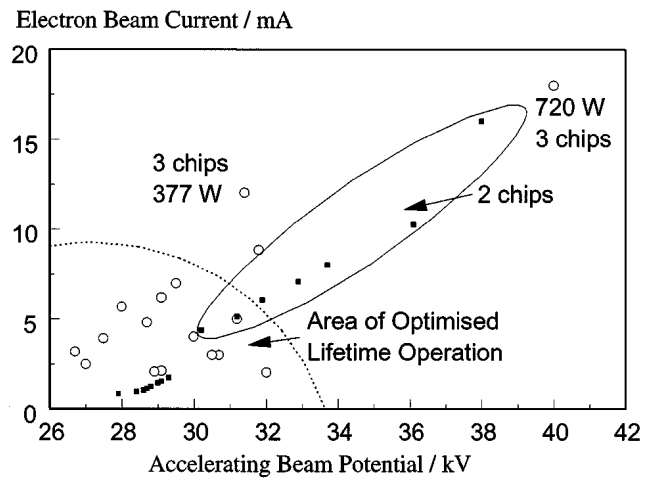

FIG. 3. Operating conditions, $I / V$, showing oscillating ( $\mathbf{a})$ and nonoscillating (o) operation. Data points refer to single chip operation unless otherwise indicated. beam tube to determine the positions at which electrons were incident upon the cavity wall. With this diagnostic, the electric and magnetic fields required for optimal beam transport through the interaction cavity were obtained.

The FEA gyrotron mm-wave output was measured using a Hewlett-Packard (HP) R8486D calibrated power meter, which was capable of measuring powers in the range $100 \mathrm{pW}$ to $1 \mathrm{~mW}$. The mm-wave output was coupled into the power detector via a variable $(0-40 \mathrm{~dB})$ in-line attenuator. At a cathode and intermediate anode potential of $-32 \mathrm{kV}$ and constant beam current of $\sim 2 \mathrm{~mA}$, the cyclotron frequency was varied by sweeping the central intracavity magnetic field. This was swept at a rate of $1 \mathrm{mT} / \mathrm{min}$ between 1.625 and $1.645 \mathrm{~T}$, and between 1.130 and $1.150 \mathrm{~T}$. Within each range, the detected $\mathrm{mm}$-wave power increased by up to 5 orders of magnitude, from $\sim 10 \mathrm{nW}$ to $\sim 1 \mathrm{~mW}$, in the ranges 1.630-1.636 and 1.138-1.144 T, Fig. 4. This represents a transition from the incoherent background cyclotron emission, verified as varying linearly with the electron beam current, to a collective emission process. The cyclotron frequencies for the magnetic fields at resonance were calculated using Eq. (1) to be 42.94 and $29.98 \mathrm{GHz}$, respectively. The gyrotron interaction frequency will be slightly greater due to a small Doppler shift, and will also be slightly above the mode cutoff frequencies of the $\mathrm{TE}_{03}$ $\left(f_{\mathrm{co}}=43.59 \mathrm{GHz}\right)$ and $\mathrm{TE}_{02}\left(f_{\mathrm{co}}=30.06 \mathrm{GHz}\right)$ modes, respectively (cavity inner diam $=22.27 \mathrm{~mm}$ ). Therefore, for frequencies closely satisfying the cyclotron resonance condition, coherent emission due to the CRM instability was observed.

The angular distribution of the mm-wave energy radiated from the output coupling horn was measured using a Ka-band crystal detector, scanned through the far-field radiation while operating conditions were kept constant. Output modes were identified by comparison with calculated mode patterns to be $\mathrm{TE}_{02}$ and $\mathrm{TE}_{01}$ for cavity fields 1.636 and $1.143 \mathrm{~T}$, respectively. It was deduced from the measured output mode patterns that mm-wave power was coupling out of the cavity via down conversion of the waveguide mode, following the pattern $\mathrm{TE}_{0 n}$ to $\mathrm{TE}_{0 n-1}$.

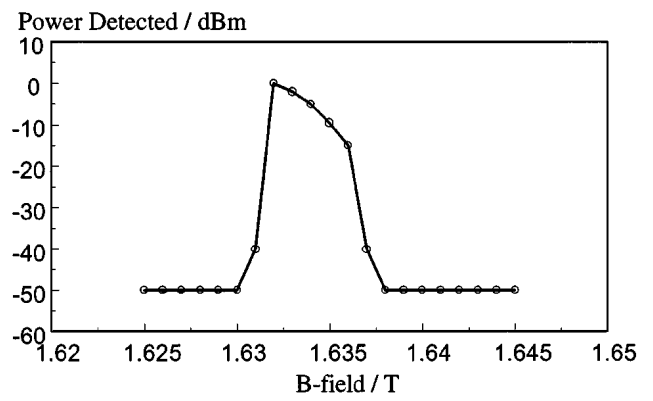

FIG. 4. Detected microwave power of the FEA gyrotron near to resonance with the $\mathrm{TE}_{03}$ operating mode. The transition to coherent CRM emission is signified by an increase in output power of 5 orders of magnitude. 
An attempt was made to operate the gyrotron in the $\mathrm{TE}_{01}$ cavity mode with cutoff frequency $16.42 \mathrm{GHz}$. However, this mode was cut off in the narrowed section of the waveguide and, since mode conversion to a lower mode with similar angular momentum was not possible, no oscillations could be observed.

Measurement of the far-field radiation power density gave an estimated $1 \mathrm{~W}$ absolute microwave output power from this gyrotron, during operation with a $100 \mathrm{~W}$ electron beam. This represents a significant conversion efficiency for a low current gyrotron of $\sim 1 \%$.

These results demonstrate that FEA cathodes can be used not only in high power electron gun designs, but also in high power mm-wave devices. Relativistic electron beams with powers of up to $720 \mathrm{~W}$ from FEAs were propagated through the gyrotron cavity and radiated coherently via the CRM mechanism. It was shown that FEA lifetimes were not unduly affected by high voltage operation, given adequate shielding. Lifetimes of the order of several hours of operation were achievable with currents of $>5 \mathrm{~mA}$ per chip under nonideal conditions.

Considering the present rate of research and device development activities throughout the world, it is likely that the next ten years should result in a number of products based on vacuum microelectronic devices. This work demonstrates one of the first such exciting applications, the operation of the first millimeter wave device employing an FEA cathode.

This work was supported by the SERC, DRA Malvern, and GEC-Marconi Ltd. Encouragement, advice and dis- cussions with N. Cade, D. F. Howell, R. D. Groves, D. M. Parkes, and K. Trafford are gratefully acknowledged.

*Present address: University of Maryland, College Park, MD 20742 and Naval Research Laboratory, Washington, DC 20375.

†Present address: Defence Research Agency, Malvern, United Kingdom.

[1] T. Utsumi, IEEE Trans. Electron Devices 38, 2276 (1991).

[2] C. A. Spindt, C. E. Holland, and R. D. Stowell, Appl. Surf. Sci. 16, 268 (1983).

[3] C. C. Curtis and K. C. Hsieh, Rev. Sci. Instrum. 57, 989 (1986).

[4] I. Brodie and C. A. Spindt, Appl. Surf. Sci. 2, 149 (1979).

[5] P. N. Lally, Y. Goren, and E. A. Nettesheim, IEEE Trans. Electron. Devices 36, 2738 (1989).

[6] C. A. Spindt, C. E. Holland, A. Rosengreen, and I. Brodie, IEEE Trans. Electron Devices 38, 2355 (1991).

[7] E. A. Adler et al., IEEE Trans. Electron Devices 38, 2304 (1991).

[8] C. A. Spindt, J. Appl. Phys. 39, 3504 (1968).

[9] A. D. R. Phelps, A.Z. Maatung, and S. N. Spark, Int. J. Electron. 64, 369 (1988).

[10] S. N. Spark and A.D. R. Phelps, J. Phys. D 22, 1807 (1989).

[11] S. N. Spark, A.D. R. Phelps, and P.R. Winning, Int. J. Infrared Millim. Waves 12, 885 (1991).

[12] A. W. Cross, Ph.D. thesis, University of Strathclyde, 1993.

[13] M. Garven, A. D. R. Phelps, and S. N. Spark, Vacuum 45, 513 (1994). 